\title{
Sequential pattern formation in a model for skin morphogenesis
}

\author{
Gerhard C. Cruywagen and P. K. Maini \\ Centre for Mathematical Biology, Mathematical Institute, 24-29 St. Giles', \\ Oxford $O X 13 L B, U K$
}

\section{J. D. Murray}

Department of Applied Mathematics FS-20, University of Washington, Seattle, Washington 98195 , US A

\author{
[Received 13 August 1992]
}

\begin{abstract}
During morphogenesis regular patterns often develop behind a frontier of pattern formation which travels across the prospective tissue. Here the authors consider the propagating patterns exhibited in a two-dimensional domain by a tissue interaction mechanochemical model for skin pattern formation. It is shown that the model can exhibit travelling waves of complex spatial pattern formation. Two alternative mechanisms that can produce such sequential patterning are presented. In particular, it is demonstrated that the specification of a simple quasi-one-dimensional pattern is all that is required to determine a complex two-dimensional pattern. Finally, the model solutions are related to actual pattern propagation during chick feather primordia initiation.
\end{abstract}

Keywords: morphogenesis; spatial patterns; tissue interaction; mechanochemical; propagating patterns, switch boundary.

\section{Introduction}

The vast range of pattern and structure observed in animals develops from the apparently homogeneous mass of cells that constitutes the early embryo. The process of pattern formation is termed morphogenesis and consists of a complex interaction of chemical, mechanical, and electrical phenomena. In this paper we shall concentrate on pattern formation in the vertebrate skin.

The skin is composed of two layers: the epidermis, consisting of sheets of columnar cells, overlies the dermis, consisting of mesenchymal cells which move on an extracellular matrix (ECM). These layers are separated by a fibrous basal lamina. Vertebrate skin forms many specialized structures, for example, hair, scales, feathers, and glands, which are distributed in a highly ordered fashion. Although widely studied experimentally, the mechanisms involved in their formation and distribution are not well understood and remain a source of much controversy. However, it is clear that tissue interaction between the dermis and epidermis plays an important role in skin organ morphogenesis (see e.g. Rawles, 1963; Dhouailly, 1973, 1975; Wessells, 1977; Dhouailly \& Maderson, 1984; Gallin et al., 1986).

Most models for morphogenesis focus on synchronous pattern formation (see Murray, 1989, for a review); however, morphogenetic processes frequently occur 
sequentially. That chick feather primordia are laid down in such a manner shall be explained below. Further examples of such propagating mechanisms of pattern formation in embryogenesis are seen in somite formation (Pearson \& Elsdale, 1979), reptilian tooth development (Edmund, 1969), scale formation (Maderson, 1965a, b), and alligator skin patterning (Murray et al., 1990; Myerscough \& Murray, 1990).

Nagorcka (1986) first proposed a tissue interaction mechanism to model the formation of feathers, scales, and hair follicles. His model consists of a pair of reacting and diffusing chemicals (termed morphogens) in the epidermis controlled by a switch mechanism in the dermis. The reaction-diffusion system produces a spatial prepattern in morphogen concentrations which provides positional information (Wolpert, 1981) for epidermal patterning and which also induces dermal cell aggregration. This model was extended by Nagorcka et al. (1987) and Shaw \& Murray (1990) to incorporate the mechanochemical interaction of dermal cells with their extracellular environment.

Recently, Cruywagen \& Murray (1992) proposed a mechanical model for tissue interaction. They demonstrated that their model could account for synchronous pattern formation in cell density. The model essentially involves mechanochemical cell movement activated by intercellular signalling. The inherent mechanical and chemical characteristics of the tissue are incorporated into the equations. Here, we investigate the sequential pattern formation properties of this tissue interaction model and apply the results to the development of feather primordia on the chick back.

Feather primordia development in the chick has been widely studied experimentally (Davidson, 1983a, b; Chuong \& Edelman, 1985a, b). The stages of feather formation are described in detail in Sengel's (1976) book. The first feather rudiments on the chick back become visible six days after egg fertilization. A feather primordium consists of an epidermal placode overlying a dermal papilla. Initially a row of equally spaced feather primordia appears along the dorsal midline. Lateral rows of feather buds are laid down sequentially from the dorsal row outwards to form a regular square-like pattern. There is no general agreement on the sequence of events in the dermis and epidermis respectively in the formation of papillae and placodes. However, there is strong evidence for dermal-epidermal coupling (Chuong \& Edelman, 1985a). We shall focus on the initial patterning stage when interaction between the dermal papillae and the epithelial placodes induces the formation of feather primordia.

In Section 2 we briefly describe the tissue interaction model and show how it can be reduced to a simpler system which still retains the essential features of the interaction mechanism. In Section 3 we present a linear analysis which delimits regions in the model parameter space where the homogeneous steady state of the model bifurcates to spatially heterogeneous solutions. We show that on a twodimensional domain the model can give rise to rhombic-type patterns which develop synchronously. In Sections 4 and 5 two different ways in which pattern formation can propagate through a domain are considered. We show that the final form of the full pattern in two dimensions can be determined by an initial quasi-one-dimensional pattern. In Section 6 the results of our model are applied to the formation of feather primordia. 


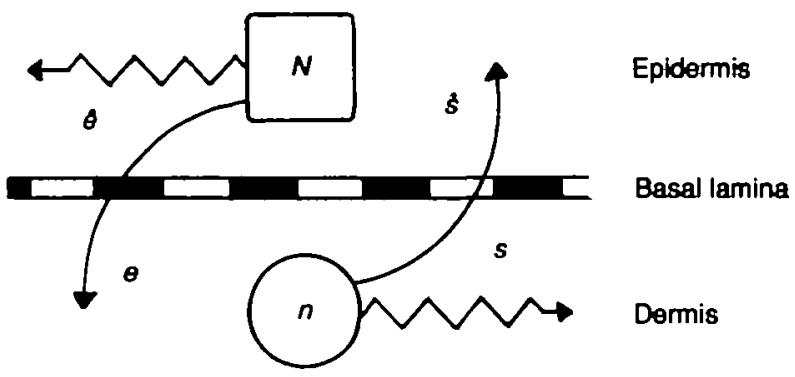

Fig. 1. A schematic diagram of the tissue interaction mechanism. The dermal cells $n$ produce a morphogen $s$, which diffuses across the basal lamina to the epidermis where it is denoted by $f$. There, the morphogen increases cell traction forces which, in turn, leads to cell aggregation and the formation of placodes. Similarly $e$, produced by the epidermal cells $N$, diffuses to the dermis where it is denoted by $e$. There, the morphogen acts as a chemoattractant for dermal cells, leading to cell aggregation and the formation of papillae.

\section{Tissue interaction model}

Here we briefly describe the epidermal-dermal tissue interaction model. A schematic diagram of the model is shown in Fig. 1. Full details may be found in Cruywagen \& Murray (1992). We begin by considering a model for epidermal morphogenesis.

We model the epithelial sheet as a two-dimensional viscoelastic continuum (see e.g. Murray \& Oster, 1984a, b; Murray, 1989). As the system is in a low Reynolds number regime, we assume that the viscoelastic and cell traction stresses within the epidermis are balanced by the external body forces. The force balance equation, then, takes the form

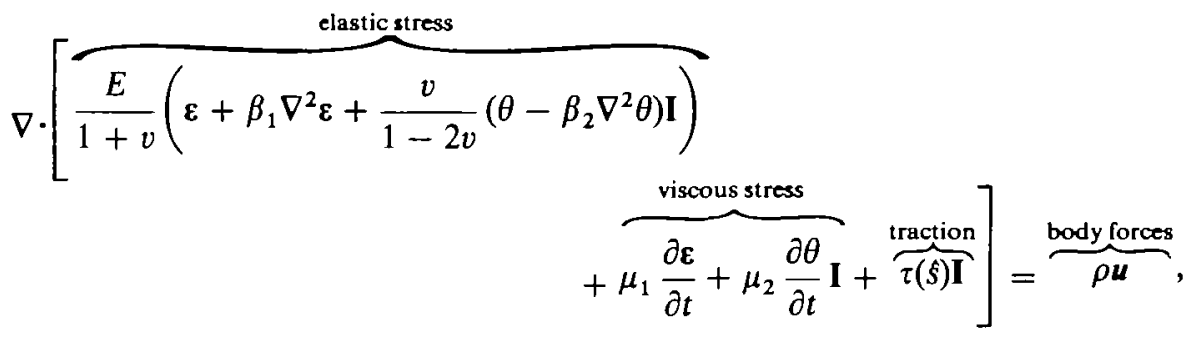

where the variable $\boldsymbol{u}(\boldsymbol{x}, t)$ is the displacement at time $t$ of a material point in the epithelial layer which was at position $x$ initially, $\theta=\nabla \cdot u$ is the dilation, $\varepsilon=\frac{1}{2}\left(\nabla \boldsymbol{u}+\nabla \boldsymbol{u}^{\top}\right)$ the strain tensor, where $T$ denotes the transpose, and $S$ is the concentration of the chemical, secreted in the dermis. The parameter $E$ is the passive elastic Young's modulus, $v$ is Poisson's ratio, $\mu_{1}$ and $\mu_{2}$ are the shear and bulk viscosities respectively (Landau \& Lifshitz, 1970), and $I$ is the unit tensor. The parameters $\beta_{1}$ and $\beta_{2}$ reflect the effect of long-range elastic stresses (see Murray, 1989, for a discussion). The epidermis is attached to the basal lamina by adhesion tethers, and the parameter $\rho$ is a measure of the strength of these attachments. All the above parameters are in fact non-negative functions of $s$ but, for simplicity, we shall assume that they are constant. 
Furthermore, the cells exert active traction which we assume depends on the chemical $\hat{s}$. We model this traction $\tau$ by the switch function

$$
\tau(\hat{s})=\frac{\tau \hat{s}^{2}(N, n)}{1+c \hat{s}^{2}(N, n)},
$$

where $\tau$ and $c$ are positive constants, and $N(x, t)$ and $n(x, t)$ are the densities of epidermal and dermal cells, respectively, at position $\boldsymbol{x}$ and time $t$ (see Murray \& Oster, 1984a).

The second equation connecting the epidermal variables $N$ and $\boldsymbol{u}$ is derived by considering cell conservation. Since the only contribution to cell flux is convection, the equation is simply

$$
\frac{\partial N}{\partial t}=\overbrace{-\nabla \cdot N \frac{\partial u}{\partial t}}^{\text {convection }} .
$$

To model dermal morphogenesis we consider a chemotaxis mode, related to the cell-chemotaxis model of Oster \& Murray (1989) and based on the morphoregulator hypothesis of Edelman (see e.g. Edelman, 1986). According to this hypothesis skin organ morphogenesis is controlled by cell-cell adhesion mechanisms mediated by cell adhesion molecules (CAMs) which function as glue to bind cells together. Because chemical modulation can have a marked effect on the binding rates and binding strengths of CAMs (Grumet \& Edelman, 1988), we assume that a chemical signal, with concentration $e$, is responsible for CAM expression. The conservation equation for dermal cell density $\mathrm{n}(\boldsymbol{x}, t)$ then takes the form

$$
\frac{\partial n}{\partial t}=\overbrace{\nabla \cdot D(e) \nabla n}^{\text {diffusion }}-\overbrace{\nabla \cdot n \nabla \alpha(e)}^{\text {chemotoxis }}+\overbrace{r n\left(n_{0}-n\right)}^{\text {mitosls }},
$$

where $D$, the coefficient of random diffusion, may be a function of $e$. As before we shall assume, for simplicity, that $D$ is constant. We have assumed that cell growth obeys the logistic law, where $r$ and $n_{0}$ are positive constants. The function $\alpha(e)$ models the chemoattraction and for the purpose of this paper we assume that it has the linear form

$$
\alpha(e)=\alpha e,
$$

where the parameter $\alpha$ is a nonnegative constant.

The interaction between epidermis and dermis is mediated through the two morphogens $s$ and $e$ which are secreted, one in each tissue, and diffuse across the membrane between the tissues. Full details may be found in Appendix $A$ and.in Cruywagen \& Murray (1992) (see Appendix B for the nondimensionalization). Here, we simply note that with the specific interaction mechanism described in Appendix $A$ we can find expressions for $\hat{s}$ and $e$ in terms of the epidermal and dermal cell densities, so that in terms of our dimensionless constants

$$
\tau(N, n)=\frac{\tau n^{2}}{(1+\nu N)^{2}+c n^{2}},
$$


and

$$
\alpha(N, n)=\frac{\alpha N}{1+\gamma n}
$$

where the parameters $\hat{v}$ and $\gamma$ are nonnegative constants.

The system $(2.1,2.3$, and 2.4$)$ constitutes the field equations of our tissue interaction model. The full model system is extremely complex but by making a few reasonable biological assumptions we can reduce it to two coupled nonlinear equations, thus making it more amenable to analysis while still retaining the essential biological features of the full model.

Firstly, we reduce the epidermal tensor equation (2.1) to a scalar dilation equation in $\theta(=\nabla \cdot u)$, by taking the divergence of both sides.

Secondly, noting that in many developmental processes the changes in cell density and in epithelial strain during pattern formation are small, we make the assumption that the dilation $\theta$ is small. With this small strain approximation, we can linearize equation (2.3) about the nondimensionalized steady state $N=1$, and after integration we get

$$
N=1-\nabla \cdot \boldsymbol{u}=1-\theta .
$$

Thus we can eliminate $N$ and reduce the system to a small-strain quasi-steady-state (SSQSS) model involving only $\theta$ and the dermal cell density $n$. The nondimensionalized SSQSS system is

$$
\begin{gathered}
\mu \frac{\partial}{\partial t} \nabla^{2} \theta+\nabla^{2} \theta-\beta \nabla^{4} \theta+\tau \nabla^{2} \frac{n^{2}}{[1+\hat{v}(1-\theta)]^{2}+c n^{2}}=\rho \theta, \\
\frac{\partial n}{\partial t}=D \nabla^{2} n-\nabla \cdot\left[\alpha n \nabla\left(\frac{1-\theta}{1+\gamma n}\right)\right]+r n(1-n),
\end{gathered}
$$

where $\mu=\mu_{1}+\mu_{2}, \beta=\beta_{1}+\beta_{2}$, and $\mu, \beta, \tau$, and $\rho$ have been divided by $(1-v) /(1-2 v)$. We also have the physical constraint that $|\theta|<1$.

In this paper we concentrate on a submodel derived by setting the parameters $\gamma$ and $\hat{v}$ equal to zero in (2.8). This amounts to ignoring the metabolism of the signal chemicals (see Appendix A). Furthermore, we shall ignore viscous effects by setting $\mu=0$ and assume that mitosis does not play an essential role in pattern formation, that is, we set $r$ equal to zero. The simpler model then has the form

$$
\begin{gathered}
\nabla^{2} \theta-\beta \nabla^{4} \theta+\nabla^{2}\left(\frac{\tau n^{2}}{1+c n^{2}}\right)=\rho \theta \\
\frac{\partial n}{\partial t}=D \nabla^{2} n-\nabla \cdot[n \nabla \alpha(1-\theta)] .
\end{gathered}
$$

This reduced caricature version retains the basic features of the tissue interaction mechanism, namely, that a high dermal cell density causes epithelial contraction, which, in turn, induces dermal cell chemotaxis. Moreover, it exhibits many of the possible patterns produced by the full model. 
This tissue we are considering can be approximated by a rectangular domain

$$
\mathscr{B}=\left\{(x, y): x \in\left[0, L_{x}\right], y \in\left[0, L_{y}\right]\right\},
$$

on which we impose boundary conditions

$$
(\boldsymbol{\eta} \cdot \nabla) n=0, \quad(\boldsymbol{\eta} \cdot \nabla) \theta=0, \quad\left(\boldsymbol{\eta} \cdot \nabla^{3}\right) \theta=0, \quad \text { for }(x, y) \text { on } \partial \mathscr{\theta},
$$

where $\eta$ is the unit normal vector on the boundary $\partial \mathscr{B}$ of the domain conditions ensure that the dermal and epidermal cell densities are conserved.

\section{Linear analysis}

Here we carry out a linear analysis of (2.9) to determine where in the parameter space the uniform steady state bifurcates to spatially heterogeneous solutions. The system admits the spatially homogeneous steady states

$$
n=0, \theta=0 \text { and } n=1, \theta=0 .
$$

For the case of spatial pattern formation the first steady state is not biologically relevant, so we ignore it and consider only the non-trivial steady state. Linearizing about this steady state gives

$$
\begin{aligned}
& \rho \theta=\nabla^{2} \theta-\beta \nabla^{4} \theta+P \nabla^{2} n, \\
& \frac{\partial n}{\partial t}=D \nabla^{2} n+\alpha \nabla^{2} \theta,
\end{aligned}
$$

where $\theta$ and $n$ now denote small perturbations from the nontrivial steady state, and

$$
P=\frac{2 \tau}{(1+c)^{2}}
$$

We look for solutions to the linear system of the form

$$
w=\left[\begin{array}{l}
\theta \\
n
\end{array}\right] \propto \mathrm{e}^{\left(i k \cdot x+\lambda\left(k^{2}\right) t\right)},
$$

where $\boldsymbol{k}$ is the wave vector, $k^{2}=\boldsymbol{k} \cdot \boldsymbol{k}$, and $\lambda\left(k^{2}\right)$ is the temporal growth rate of the disturbance. Substituting $w$ into the linearized system (3.1) leads to the dispersion relation

$$
\lambda\left(k^{2}\right)=-c\left(k^{2}\right) / b\left(k^{2}\right)
$$

where

$$
b\left(k^{2}\right)=\beta k^{4}+k^{2}+\rho, \quad c\left(k^{2}\right)=\beta D k^{6}-(P \alpha-D) k^{4}+\rho D k^{2} .
$$

The uniform steady state is linearly unstable to spatially varying disturbances if $\Re \lambda(0) \leqslant 0$, and $\Re \lambda\left(k^{2}\right)>0$ for a range of nonzero $k^{2}$. From (3.3) it is clear that the uniform steady state is linearly unstable if and only if $c\left(k^{2}\right)<0$ for some eigenvalue $k^{2}$. The forms of $c\left(k^{2}\right)$ and $\lambda\left(k^{2}\right)$ are sketched in Fig. 2. With a dispersion relation of this form, one can easily select and isolate different unstable eigenvalues. From the 


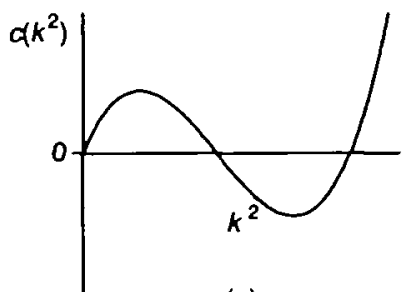

(a)

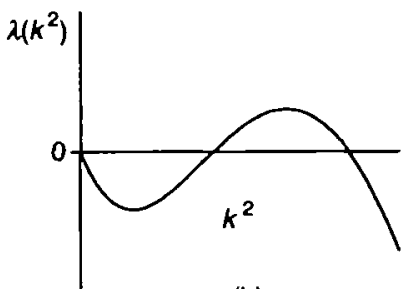

(b)

FIG. 2. The dispersion relation (3.3) for the reduced caricature tissue interaction model (2.9): (a) sketch of the function $c\left(k^{2}\right)$, (b) sketch of the dispersion relation $\lambda\left(k^{2}\right)$. Linear analysis predicts that the uniform steady state $n=1, \theta=0$ of the model equations is unstable to eigenvalues $k^{2}$ with $\Re \lambda\left(k^{2}\right)>0$.

expression for $c\left(k^{2}\right)$ it is clear that one can use any parameter as the bifurcation parameter; for illustrative purposes we shall focus on $\rho$.

It is easy to show that if $P \alpha-D>0$ then the uniform steady state loses stability as $\rho$ decreases below the critical value $\rho_{\mathrm{c}}$ which satisfies $\rho_{\mathrm{c}}=(P \alpha-D)^{2} / 4 \beta D^{2}$. The critical eigenvalue that goes unstable at this point is $k_{\mathrm{c}}^{2}=\left(\rho_{\mathrm{c}} / \beta\right)^{1 / 2}$.

So far we have assumed that the eigenvalue $k^{2}$ is continuous. However, the zero-flux boundary conditions constrain $k^{2}$ to discrete values. In particular, solutions to the linear system (3.1), satisfying the boundary conditions (2.10b), are

$$
\boldsymbol{w}(\boldsymbol{x}, t)=\left[\begin{array}{l}
\theta \\
n
\end{array}\right]=\sum_{\phi, \psi} p(\tilde{\phi}, \tilde{\psi})\left[\begin{array}{c}
1 \\
M\left(k^{2}\right)
\end{array}\right] \mathrm{e}^{\lambda\left(k^{2}\right) t} \cos \phi x \cos \psi y,
$$

where

$$
M\left(k^{2}\right)=\frac{-\alpha k^{2}}{\lambda\left(k^{2}\right)+D k^{2}} .
$$

The wave vectors $\boldsymbol{k}$ are now discrete and take the form $\boldsymbol{k}=[\phi, \psi]^{\top}$, where

$$
\phi=\tilde{\phi} \pi / L_{x}, \quad \psi=\tilde{\psi} \pi / L_{y} \quad(\tilde{\phi}, \tilde{\psi}=0,1,2, \ldots)
$$

and $k^{2}=\phi^{2}+\psi^{2}$. Each $p(\tilde{\phi}, \tilde{\psi})$ is determined by a Fourier transform of the initial conditions in terms of

$$
\left[\begin{array}{c}
1 \\
M\left(k^{2}\right)
\end{array}\right] \cos \phi x \cos \psi y .
$$

The spatially heterogeneous solution that emerges for large time for the linear system is the sum of the modes

$$
p(\tilde{\phi}, \tilde{\psi})\left[\begin{array}{c}
1 \\
M\left(k^{2}\right)
\end{array}\right] \cos \phi x \cos \psi y,
$$

corresponding to the mode pairs $(\tilde{\phi}, \tilde{\psi})$ for which

$$
\lambda\left(\left(\frac{\pi \tilde{\phi}}{L_{x}}\right)^{2}+\left(\frac{\pi \tilde{\psi}}{L_{y}}\right)^{2}\right)>0
$$


We now examine the case in which only one of the discrete eigenvalues, say $k_{\mathrm{c}}^{2}$, is unstable, that is, we assume that the parameters are such that this eigenvalue is isolated. Depending on the domain geometry, this could be either a simple or a multiple eigenvalue. In the case of a multiple eigenvalue, more than one of the mode pairs $(\tilde{\phi}, \tilde{\psi})$, from the above sequence (3.4), satisfy the expression

$$
k_{\mathrm{c}}^{2}=\left(\frac{\tilde{\phi} \pi}{L_{x}}\right)^{2}+\left(\frac{\tilde{\psi} \pi}{L_{y}}\right)^{2} .
$$

Here, we only discuss briefly the synchronous pattern-forming properties of general models of the form (2.8) at bifurcation from a simple eigenvalue. We illustrate our results by looking at the solutions to the steady-state problem for the epithelial dilation, $\theta$. The dilation variable $\theta$ differs from $n$ by the constant factor $1 / M\left(k_{\mathrm{c}}^{2}\right)$. In general, solutions corresponding to single mode pairs give rise to rhombic patterns. The linear time-independent solution which will grow is

$$
\theta(\boldsymbol{x})=\cos \phi x \cos \psi y,
$$

where $\boldsymbol{k}_{\mathrm{c}}=[\phi, \psi]^{\top}$ is the corresponding wave vector satisfying the zero-flux boundary conditions (2.10b). Without loss of generality we assume $\theta(0,0)=0$.

The solution (3.5) can be rewritten in polar coordinates $(r, \vartheta)$ (see e.g. Murray, 1989) as

$$
\theta(r, \vartheta)=\frac{1}{2}\{\cos [r(\phi \cos \vartheta+\psi \sin \vartheta)]+\cos [r(\phi \cos \vartheta-\psi \sin \vartheta)]\} .
$$

By considering $(\phi, \psi)$ as variables and transforming them to polar coordinates $\left(\kappa, \frac{1}{2} \varphi\right)$, one can write expression (3.6) as

where

$$
\theta(r, \vartheta)=\frac{1}{2}\left\{\cos \left[\kappa r \cos \left(\vartheta-\frac{1}{2} \varphi\right)+\cos \left[\kappa r \cos \left(\vartheta+\frac{1}{2} \varphi\right)\right]\right\}\right.
$$

$$
\kappa=\left(\phi^{2}+\psi^{2}\right)^{1 / 2}=\left(k_{\mathrm{c}}^{2}\right)^{1 / 2} \text { and } \varphi=2 \arccos \left[\phi /\left(k_{\mathrm{c}}^{2}\right)^{1 / 2}\right]
$$

It is now easy to see from expression (3.7) that $\varphi$ represents the rhombic angle.

This solution is invariant under a rhombic rotation, that is

$$
\theta(r, \vartheta)=\theta(r, \vartheta+\pi)=R \theta(r, \vartheta)=\theta(r, \vartheta)
$$

where $R$ is the rhombic operator.

An illustrative example of a rhombic pattern corresponding to the mode pair $(6,2)$ on a square domain $(1,1)$ is presented in Fig. 3. The unstable wave vector here is $\mathbf{k}_{\mathrm{c}}=[\phi, \psi]^{\top}=[6 \pi, 2 \pi]^{\top}$ and so the rhombic angle of the pattern is $\varphi=\frac{1}{6} \pi$.

Note that if $\varphi=\frac{1}{2} \pi$ or $\frac{3}{2} \pi$, then $\phi=\psi$ and we get a square- or chessboard-type pattern which is a special case of the rhombic pattern. In this case

$$
\theta(r, \vartheta)=\frac{1}{2}[\cos (r \kappa \cos \vartheta)+\cos (r \kappa \sin \vartheta)]
$$

with $\kappa$ as in (3.8). This solution is square rotationally invariant since

$$
\theta(r, \vartheta)=\theta\left(r, \vartheta+\frac{1}{2} \pi\right)=S \theta(r, \vartheta)=\theta(r, \vartheta)
$$

where $S$ is the square rotational operator. 


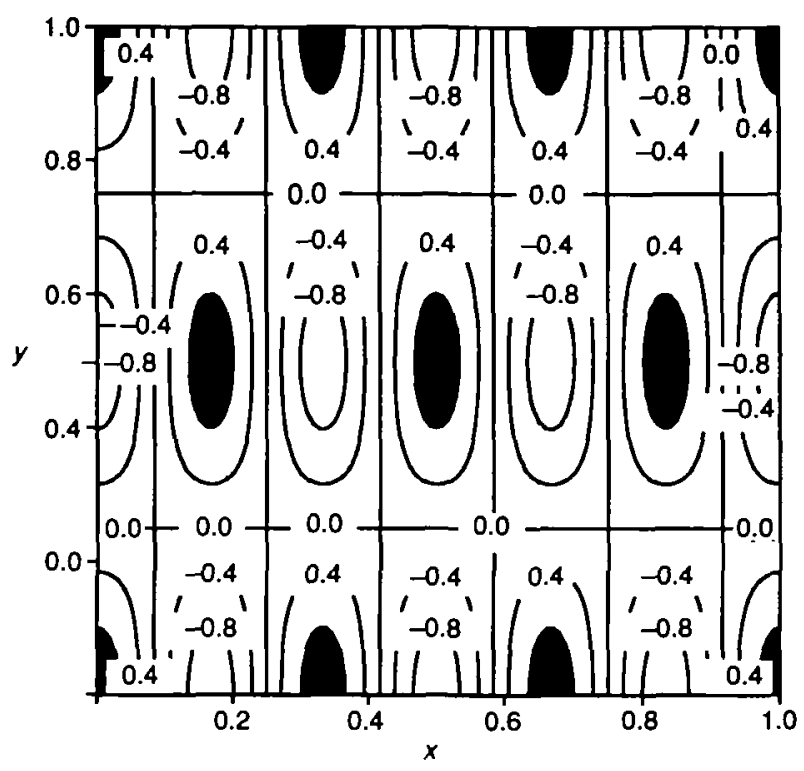

FIG. 3. An illustrative example of a linear rhombic pattern corresponding to the mode pair $(6,2)$ on a square domain $(1,1)$. Regions of high epithelial dilation $(\theta>0.8)$ are shaded.

The simplest pattern we can get on the two-dimensional rectangular domain is the roll, which occurs when either $\phi=0$ or $\psi=0$. The roll is invariant under a rotation of $\pi$.

All of the solutions discussed above satisfy

$$
\Gamma\left(x+j \omega_{1}+l \omega_{2}\right)=\Gamma(x),
$$

where $\Gamma=(n, \theta)$ is the solution of the system, $\boldsymbol{x}$ is an arbitrary vector, $j$ and $l$ are integers, and $\omega_{1}$ and $\omega_{2}$ are appropriately chosen independent vectors. The solutions are therefore regular tessellations of the plane.

Naturally, in cases where we have a degenerate eigenvalue, there is a much richer and more complicated range of patterns possible.

In all the above cases linear analysis gives us only a very rough guide as to the patterns we should expect. To investigate the problem fully, numerical simulation of the full nonlinear system (2.9) is necessary.

\section{Sequential pattern formation from initial pattern}

We examine in this section the ability of an initial given pattern to propagate through a two-dimensional domain. This could give an indication as to which mechanism(s) actually operate in skin morphogenesis.

As we saw in the previous section the linearized two-dimensional problem with zero-flux boundary conditions only admits a discrete set of unstable modes. As before, we assume there that we are close enough to the bifurcation point to spatial pattern so that $k_{\mathrm{c}}^{2}$ is the only unstable eigenvalue. 
Since the wave vectors corresponding to the unstable eigenvalue

$$
k_{\mathrm{c}}^{2}=\phi^{2}+\psi^{2} \text {, }
$$

are of the form $\boldsymbol{k}_{\mathrm{c}}=[\phi, \psi]^{\top}$, each wave vector has in fact two degrees of freedom. On an infinite domain any combination $[\phi, \psi]^{\top}$ satisfying (4.1) could be selected, depending on the initial conditions. However, on a finite domain, the values which $\phi$ and $\psi$ can take are determined by the size and shape of the domain as well as the boundary conditions.

We solve the system on the domain $\mathscr{B}$, see (2.10a), with $L_{y} \ll L_{x}$, so that the domain is much larger in the $x$ - than in the $y$-direction. Initially it is assumed that the system is at the homogeneous steady state $\theta=0, n=1$ everywhere except for a small subdomain at one end

$$
\mathscr{B}^{*}=\left\{(x, y): 0 \leqslant x \leqslant L_{x}^{*}, 0 \leqslant y \leqslant L_{y}\right\},
$$

where $\mathrm{L}_{x}^{*} \ll \mathrm{L}_{x}$. We specify an initial pattern in this small region and examine its propagation across the rest of the domain. How the initial pattern is set up does not concern us for the moment. Because of the zero-flux boundary conditions at $y=0$ and $y=L_{y}$ the $y$-component $\psi$ of the wave vector $\boldsymbol{k}_{\mathrm{c}}$ of the developing pattern, is necessarily fixed by the initial pattern. The $x$-component $\phi$ of the spreading pattern is thus forced to take on a certain value so as to satisfy (4.1).

We now show two numerical examples to illustrate the different types of travelling waves of pattern formation that can arise. In both examples a rectangular domain of dimensions $=\left(6 \sqrt{ } 2, \frac{3}{2} \sqrt{ } 2\right)$ is considered, so $L_{y} \ll L_{x}$. From the linear analysis we choose parameters

$$
\tau=4.0, \quad c=1.0, \quad \rho=19.739, \quad \beta=0.012665, \quad \alpha=2.0, \quad D=2.0,
$$

so that the unstable eigenvalue is $k_{\mathrm{c}}^{2}=4 \pi^{2}$.

Example 4.1 As initial conditions we specify a pattern satisfying the zero-flux boundary conditions (2.10), corresponding to the wave vector $\boldsymbol{k}_{\mathrm{c}}=[2 \pi, 0]^{\top}$ on the subdomain $\mathscr{B}^{*}$ with $L_{x}^{*}=\frac{3}{4}$. The homogeneous steady state is specified everywhere else (see Fig. 4(a)). As one might expect, this stripe pattern propagates along the length of the domain, as can be seen in Fig. 4(b, c).

Example 4.2 Next we set as initial conditions a pattern resembling the wave vector $\boldsymbol{k}_{\mathrm{c}}=[2 \pi / \sqrt{ } 2,2 \pi / \sqrt{ } 2]^{\top}$ on the subdomain $\mathscr{B}^{*}$, where $L_{x}^{*}=\frac{1}{4} \sqrt{ } 2$. Thus the first half row of pattern in the $x$-direction is specified (see Fig. 5(a)). As was the case with the stripe pattern in the previous example, we see that new rows are added progressively in the $x$-direction, which leads, in this case, to a rhombic pattern (see Fig. 5(b, c)).

An important point, illustrated by these numerical simulations, is that an initial pattern specified on a small subdomain determines the form of the evolving propagating pattern. Also, a simple quasi-one-dimensional initial pattern is all that is required to specify the complicated pattern over the whole two-dimensional domain.

Both the above examples resemble real pattern formation processes seen in embryology. The first simulation is similar to the stripe pattern which is laid down 
MODEL FOR SKIN MORPHOGENESIS

237
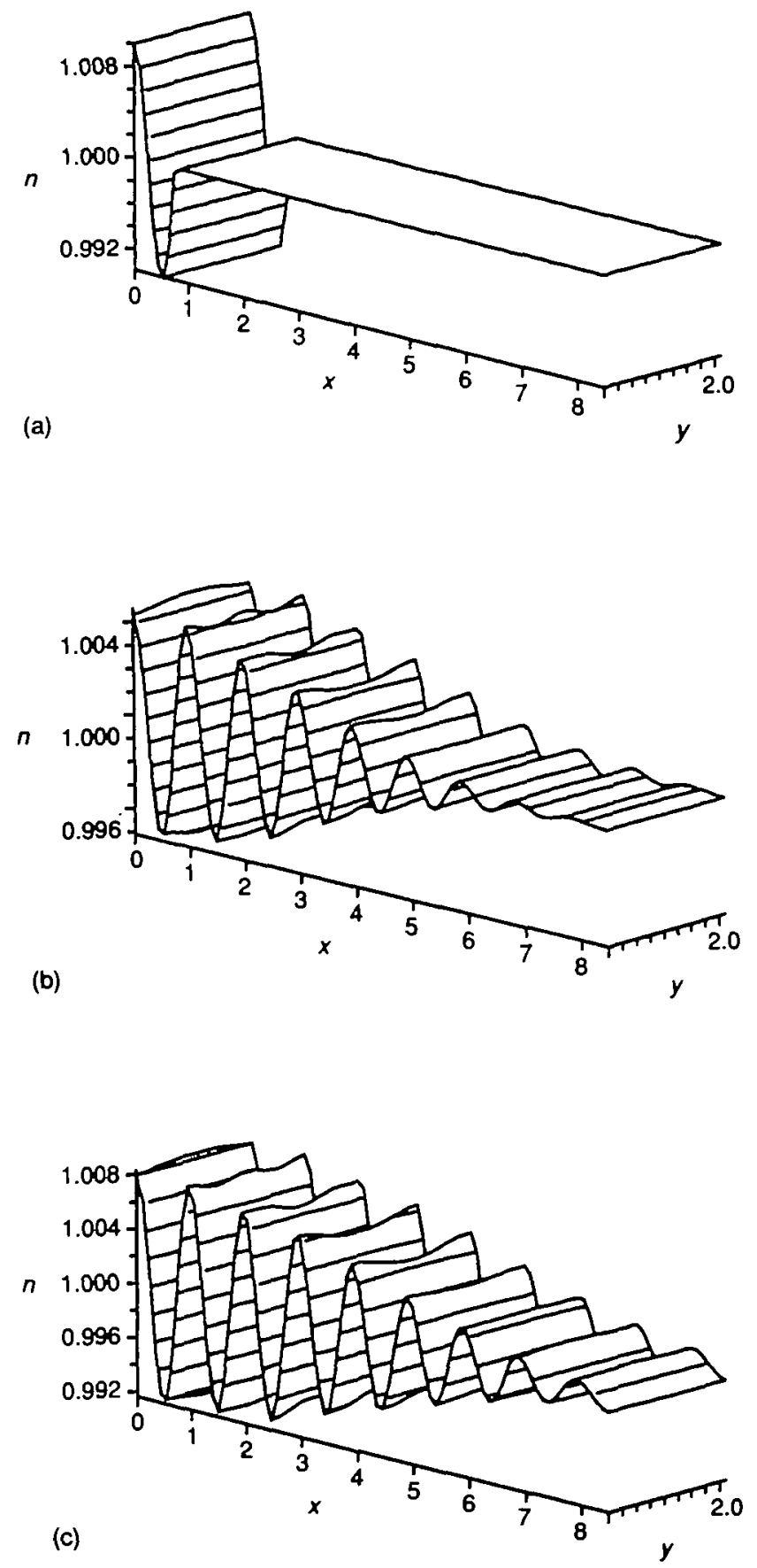

FIG. 4. Sequential stripe formation on a rectangular domain for the caricature tissue interaction model (2.9) (see Example 4.1 for details). (a) Initially a single stripe is specified on the left of the rectangular domain. As the system evolves, the stripes propagate along the domain; (b) and (c) show cell density profiles at $t=5.0$ and $t=10.0$ respectively. 

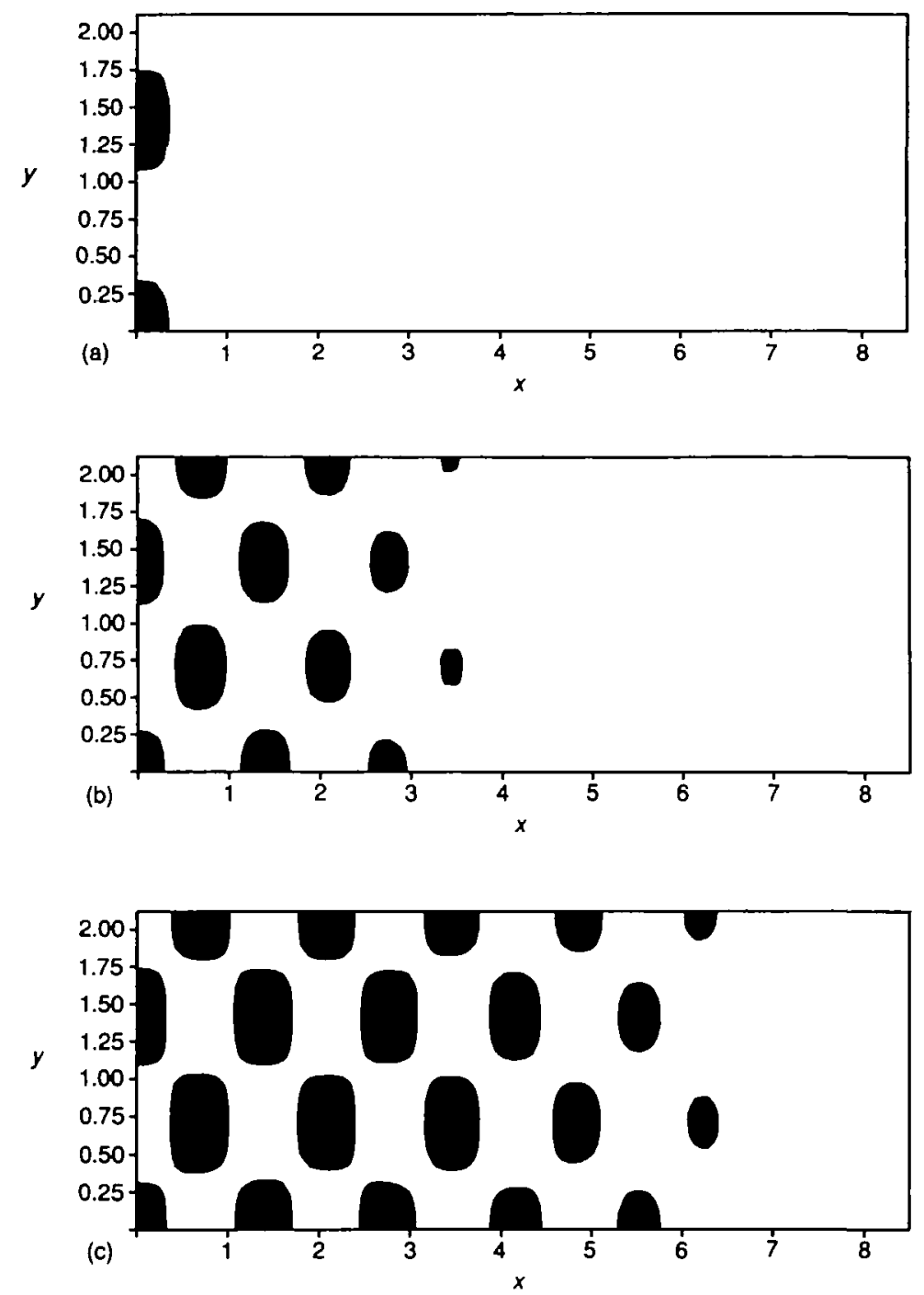

FIG. 5. Sequential pattern formation in the caricature model (2.9) for tissue interaction (see Example 4.2 for details). Regions of high cell density ( $n>1.0$ ) are shaded. (a) Initially a single row is specified at one end of a rectangular domain. As the system evolves, the pattern propagates along the domain with more rows added sequentially; (b) and (c) show cell density profiles at $t=3.0$ and $t=6.0$ respectively.

sequentially on the alligator embryo. The second simulation mimics the sequential row-by-row feather bud formation on the chick back. Note that we are only concerned here with transient states and that when the pattern reaches the boundary $x=L_{x}$ it may rearrange to form a final steady-state pattern. However, in biological tissue such as a rearrangement is irrelevant since cells only have a small window of time in which they can respond to their environment before the pattern is locked in.

Depending on the basin of attraction of each pattern, random initial perturbations 
could develop into any of these two propagating patterns and so a specific pattern cannot be set up by the natural inhomogeneities present in the embryonic tissue. For the above propagating mechanism to operate, the initial pattern has to be specified independently of the model mechanism.

\section{A switch mechanism in sequential pattern formation}

In the previous section we illustrate one possible scenario to account for the propagation of spatial pattern across a domain. In this section we present an alternative scenario in which a temporal mechanism propagates across the tissue making it responsive to the spatial patterning mechanism. Such a temporal wave may be thought of as switching on tissue to pattern formation. Nagorcka (1986) first introduced the concept of a temporal wave of determination in a reaction-diffusion model for pattern formation.

His model consists of a dermal switch mechanism controlling the epidermal morphogenesis. At any time during the pattern formation process, a line, which Nagorcka (1986) calls a switch boundary, could be drawn dividing a region of tissue where the pattern has developed, from where it still has to develop. He introduced such a switch boundary in his model by assuming that the dermal switch mechanism has switched on the epidermal pattern formation process inside the boundary, while outside the boundary pattern formation remains switched off. We introduce a similar switch boundary to our model.

We use as switch boundary a line satisfying the parametric equation $x=v t$, where $v$ is the speed of propagation. We assume $v$ is positive so that the switch boundary moves in the positive $x$-direction away from $x=0$ as time increases from $t=0$. As before the zero-flux boundary conditions (2.10b) are used.

We further assume that the switch mechanism resides in the dermis. It could, for example, be due to a change in production of the dermally produced chemical morphogen. In terms of our dimensionless parameters this would be reflected by a switch in $\tau$. Alternatively, it may be due to a change in the chemotactic response of the dermal cells to the signal chemical received from the epidermis, which would be reflected by a switch in the parameter $\alpha$. Since a decrease in the strength of the tethering of the pithelial layer through the basal lamina into the dermal layer causes pattern formation, one could also use the tethering parameter $\rho$ as a switch. At present, there is no good biological evidence for favouring any particular switch mechanism. Note that any of the parameters in the reduced small strain quasi-steadystate tissue interaction model can be used as a switch or bifurcation parameter.

For illustrative purposes we select $\rho$ as bifurcation parameter and set

$$
\rho(x, t)=\left\{\begin{array}{ll}
\rho_{1} & \text { if } x \leqslant v t \\
\rho_{2} & \text { if } x>v t
\end{array} \text { where } \rho_{1}<\rho_{\mathrm{c}}<\rho_{2},\right.
$$

in which $\rho_{\mathrm{c}}$ is the critical value where the system bifurcates from a homogeneous steady state to patterned solutions. So, the uniform steady state is linearly unstable behind the switch boundary, but linearly stable beyond it. For notational convenience we shall denote the switch boundary by $\tilde{L}_{x}$ and the domain inside the switch 
boundary by

$$
\tilde{\mathscr{B}}=\left\{(x, y): 0 \leqslant x \leqslant \tilde{L}_{x}, 0 \leqslant y \leqslant L_{y}\right\} .
$$

We assume that $\rho_{1}$ is such that there is only one unstable eigenvalue, say $k_{\mathrm{c}}^{2}$. This distinguishes our work from that of Nagorcka (1986), who had a range of unstable eigenvalues. As initial conditions we specify small random perturbations about the homogeneous steady state across the whole domain.

So as to get an intuitive understanding of the problem we assume that our developing solution in the domain $\tilde{\mathscr{B}}$ can be expressed in the linear form

$$
\left[\begin{array}{l}
\theta \\
n
\end{array}\right]=\left[\begin{array}{l}
0 \\
1
\end{array}\right]+\left[\begin{array}{c}
1 \\
M\left(k_{\mathrm{c}}\right)
\end{array}\right] \mathrm{e}^{\lambda\left(k_{\mathrm{c}}^{2}\right) t} f\left(x, \tilde{L}_{x}\right) \cos \psi y,
$$

where $M\left(k_{\mathrm{c}}\right)$ is as in the linear analysis of Section $3, \lambda$ is the dispersion relation (3.3), and

$$
\psi=\tilde{\psi} \pi / L_{y} \leqslant k_{\mathrm{c}}^{2} \quad \text { where } \tilde{\psi}=0,1,2, \ldots .
$$

Note that this expression satisfies the zero-flux boundary conditions (2.10b) at $y=0$ and $y=L_{y}$. We assume that the function $f\left(x, \tilde{L}_{x}\right)$ is such that it satisfies the zero-flux boundary conditions at $x=0$ and also at $L_{x}$ when $\widetilde{L}_{x}$ eventually reaches $L_{x}$.

Two numerical examples are now considered and in both we use the same parameters as in Example 4.1 Recall that the unstable eigenvalue is $k_{\mathrm{c}}^{2}=4 \pi^{2}$. We choose the switch parameter values as $\rho_{1}=18.239$ and $\rho_{2}=21.239$.

Example 5.1 In the first example the width of the domain is chosen as $L_{y}=2.0$, while the length $L_{x}=6 \sqrt{2}$, so that $L_{y} \ll L_{x}$. Various values for the speed $v$ of the travelling switch boundary were used. In all the cases its value was taken to be very small relative to the rate of pattern formation.

As the switch boundary moves away from $x=0$ a striped pattern perpendicular to the switch boundary is laid down, as can be seen in Fig. 6. The developing solution adheres to the form of the expression (5.1) and in this case the value of $\tilde{\psi}=4$, which is the maximum value that it can assume. Also when $\tilde{L}_{x}$ hits the boundary $L_{x}$, the value of $f\left(x, \tilde{L}_{x}\right)=1$, which would be the solution predicted by a linear analysis on the whole domain $\mathscr{B}$.

It is important to note here that as the switch boundary moves out it selects, in terms of our approximate expression (5.1), the pattern with the highest integral mode number possible that can fit into the $y$-direction, that is, a value $\psi=4$, rather than say $3,2,1$, or even zero.

We now examine a second example where the pattern that develops is such that $f\left(x, \widetilde{L}_{x}\right) \neq 1$ for any $\widetilde{L}_{x}=L_{x}$.

Example 5.2 Here we choose our domain length as before, but now choose the width as $L_{y}=1.2 \sqrt{ } 2$, so that the largest integral mode that can fit into the $y$-direction has mode number 3 . As in Example 5.1, as the switch moves away from $x=0$, stripes perpendicular to the switch boundary develop, but now $\tilde{\psi}=3$ instead of 4 . However, these stripes do not persist, and, as the switch continues across the domain, they break up to form a rhombic pattern as can be observed in Fig. 7. Again the system of equations was solved for different speeds of the moving switch boundary, but the same qualitative result was obtained in each case. 


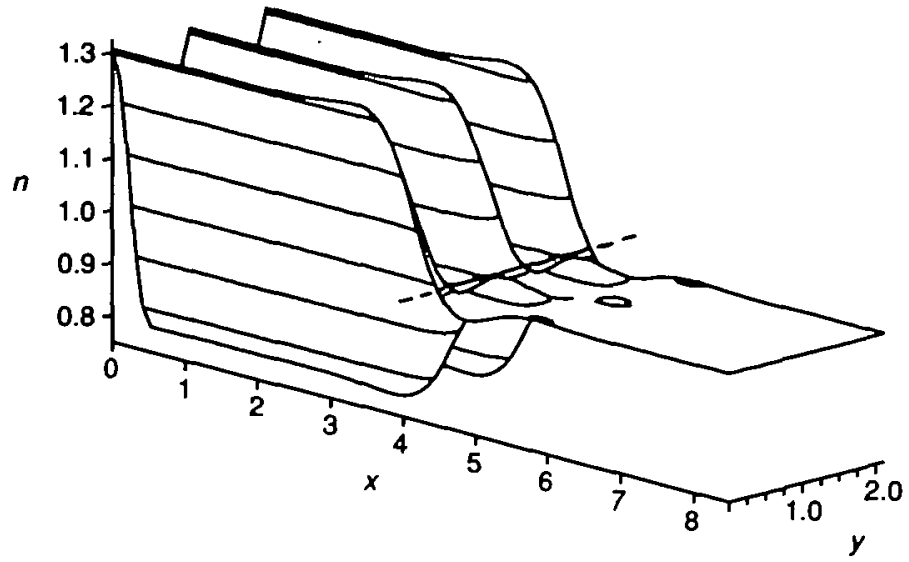

Fig. 6. Cell density profiles for the case of sequential pattern formation in the reduced caricature tissue interaction model (2.9) with a moving switch mechanism indicated by the broken line (see Example 5.1 for details). Initial conditions are random perturbations about the nontrivial homogeneous steady state specified over the whole domain.

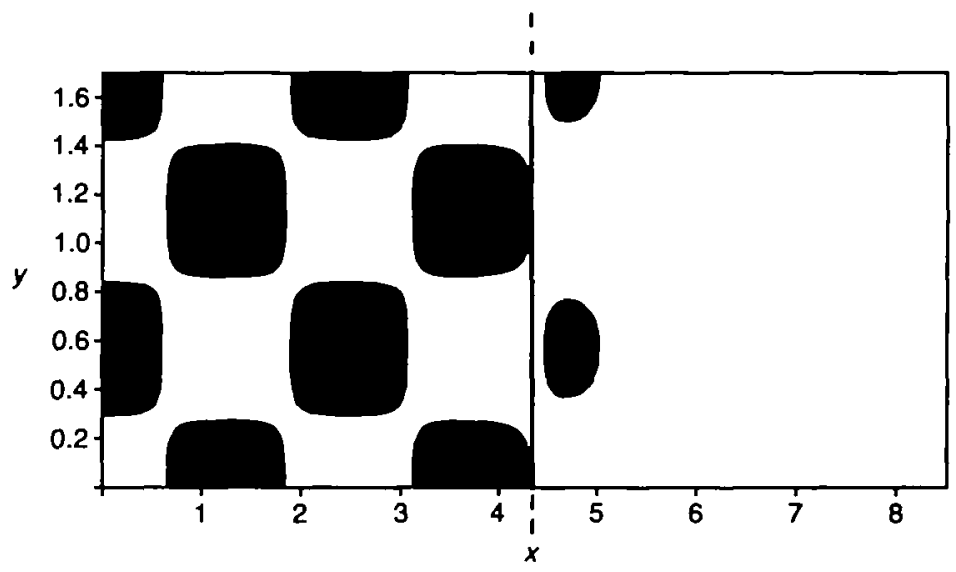

FiG. 7. Sequential pattern formation in the reduced caricature tissue interaction model (2.9) with a moving switch mechanism indicated by the vertical line (see Example 5.2 for details). Initial conditions are random perturbations about the nontrivial homogeneous steady state specified over the whole domain. Regions of high cell density $(n>1.0)$ are shaded.

Why do the stripes break up into a rhombic pattern, whereas in the previous example they persisted? This is because, in terms of our linear approximation (5.1), the value of the $y$-component $\psi$ of the wave vector $\boldsymbol{k}_{\mathrm{c}}=[\phi, \psi]^{\top}$ corresponding to the developing pattern is such that the $x$-component $\phi$ is forced to be nonzero so as to satisfy expression (4.1).

For the purpose of our explanation, we assume that $L_{x}=4 \sqrt{2}$. Thus when $\tilde{L}_{x}$ reaches $L_{x}$, to satisfy expression (4.1), $\tilde{\phi}$ must take the value 3 . Thus the pattern which develops on this rectangular domain has the wave vector $k_{c}=\left[3 \pi / L_{x}, 3 \pi / L_{y}\right]^{\top}$, which is also the wave vector that the linear analysis on this domain would predict. 
We computed several examples with different domain sizes and parameter values and found in all the cases that as the switch boundary travels away from the origin, the pattern that forms is such that $\tilde{\psi}$ takes the highest integer value that satisfies the constraint (4.1). So it seems that the pattern corresponding to the wave vector with the largest possible $y$-component always develops.

To simulate the typical chess-board-type pattern of primordia, as observed on the chick back, we must therefore choose our domain width $L_{y}$ appropriately. When $\tilde{L}_{x}$ hits $L_{x}$ we require the unstable wave vector $\boldsymbol{k}_{\mathrm{c}}=[\phi, \psi]^{\top}$ to be such that it specifies a square pattern.

Without loss of generality, we can assume that $L_{x}=L_{y}$. Hence,

$$
\phi=\tilde{\phi} \pi / L_{y}, \quad \psi=\tilde{\psi} \pi / L_{y} \quad \text { where } \quad \tilde{\phi}, \tilde{\psi}=0,1,2, \ldots .
$$

We now calculate for which value of $L_{y}$ the developing pattern's wave vector is such that $\tilde{\phi}=\tilde{\psi}$, which is the condition for a square pattern on a square domain.

As we have discussed above, the pattern which develops is the one with the highest possible integral mode that can fit into the $y$-direction. So that a pattern corresponding to the wave vector with the $y$-component $(\tilde{\psi}+1) \pi / L_{y}$ cannot fit into the specified domain, we require that

$$
(\tilde{\psi}+1)^{2} \pi^{2} / L_{y}^{2}>k_{\mathrm{c}}^{2} .
$$

Also, from (4) it follows that

$$
L_{y}^{2}=\left(\tilde{\phi}^{2}+\tilde{\psi}^{2}\right) \pi^{2} / k_{\mathbf{c}}^{2}
$$

Combining (5.2) and (5.3) we get the inequality

$$
2 \tilde{\psi}+1-\tilde{\phi}^{2}>0
$$

Thus the required condition for square patterns, namely $\tilde{\psi}=\tilde{\phi}$, is satisfied only if $\tilde{\psi}=1,2$. So only chessboard patterns corresponding to the mode pairs $(1,1)$ and $(2,2)$ are possible. If $\tilde{\psi}>2$ we are forced to choose $\tilde{\phi}$ much smaller than $\tilde{\psi}$ so as to satisfy inequality (5.4). In such cases rhombic patterns, as were shown in the previous example, will develop.

From the discussion above we saw that the chessboard arrangement of primordia, as seen on the chick back, can develop only for extremely narrow domains, that is, if the length of the initial row is less than or equal to two primordia. For larger domains, as in Example 5.2, a rhombic pattern will develop with the long sides of the rhombi perpendicular to the switch line.

\section{Biology of sequential pattern formation}

We now consider the application of the model to the feather primordia formation on the chick back. The above simulations show that there are two possible ways to explain this phenomenon in terms of our tissue interaction model.

In the first, the complex two-dimensional rhombic pattern is determined by a travelling wave of pattern formation evolving from an appropriate one-dimensional pattern specified on a portion of the dorsal midline. Biologically this method has the drawback that an initial pattern must be specified externally from the model mechanisms. 
In the second scenario, the pattern precedes a parameter-specifying wavefront sweeping across the domain. As we have seen, the domain width is extremely crucial for obtained the required pattern. The fact that rhombic patterns will develop from an initial row rather than the required square patterns is not a major problem. In his simulations of reaction-diffusion systems, Nagorcka (1986) also got such rhombic patterns, but for different reasons. However, because the growth rate of the chick embryo is very rapid in the longitudinal direction compared to the lateral growth rate, these rhombic patterns could be stretched into the observed square-like patterns.

Also, the type of laterally stretched pattern obtained in Example 5.2 is reminiscent of the observed arrangement of scales in a number of lizard species (see Cogger, 1975). Since the temporal formation of scales in lizards is similar to that of feather buds in the chicken (Maderson, 1965a), a similar travelling switch boundary could therefore operate in lizard scale formation.

Both the above mechanisms give rise to patterns that are consistent with those observed during normal development. In particular, both predict that the initial quasi-one-dimensional pattern determines the form of the two-dimensional propagating pattern. This agrees with the experimental results of Davidson (1983a, b). He cut chick skin parallel to the dorsal midline and at various distances ahead of the developing feather primordia, and compared the resulting patterns with those on the control skin, which was uncut. He found that the row immediately beyond the cut was slightly shifted (away from the dorsal midline) according to the position of the cut. The following rows, however, developed in their correct positions in relation to the first row beyond the cut. The time delay in the development of the first row beyond the cut differed by less than the interval between the development of successive rows.

These experiments suggest that the spatial arrangement of the first row of primordia that develops, whether on the dorsal midline in the case of intact tissue, or immediately beyond the cut in the case of excised tissues, determines the subsequent position of primordia and hence specifies the full two-dimensional pattern.

Therefore, it appears that the problem of generating a complex two-dimensional pattern can be reduced to that of generating a simple one-dimensional pattern. In the context of our model, the initial pattern could either be set up by a specially designated mechanism or can develop due to a spatio-temporal gradient in some parameter.

\section{Discussion}

In this paper we have investigated a caricature tissue interaction model for propagating pattern formation in two dimensions. We have shown that a complex two-dimensional spatial pattern can be determined by specifying a simple onedimensional pattern.

As a particular biological application we considered feather primordia initiation on the chick back. Two mechanisms for describing this process were proposed. We saw that a periodic spatial pattern simulating the initial row along the dorsal midline can determine the resulting two-dimensional chessboard pattern on the chick. Our model simulations thus agree well with the experimental results of Davidson $(1983 \mathrm{a}, \mathrm{b})$.

Davidson's results also clearly demonstrate that feathers do not develop in 
predefined positions. For example, by stretching or cutting the skin one can influence the positions in which the buds develop. Hence the wave of feather determination is not a prepattern wave which determines the positions of the feather buds through positional information, rather it enables areas of tissue to become competent for primordia formation. This experiment also indicates that chick skin pattern formation is a process where the mechanical and chemical properties of the skin, combined with geometrical aspects, play a crucial role.

\section{Appendix A}

\section{The tissue interaction mechanism}

Full details of the tissue interaction model may be found in Cruywagen \& Murray (1992). Here we focus on the interaction mechanism which determines the functional form of $s$ and $e$ in (2.1) and (2.4), respectively, in terms of cell densities $n$ and $N$. We define

$$
\begin{aligned}
\hat{e}(x, t)= & \text { the epidermal concentration of the signal morphogen, produced in } \\
& \text { the epidermis, at position } x \text { and time } t ; \\
s(x, t)= & \text { the dermal concentration of the signal morphogen produced in the } \\
& \text { dermis; } \\
e(x, t)= & \text { the dermal concentration of the signal morphogen received from the } \\
& \text { epidermis. } \\
s(x, t)= & \text { the epidermal concentration of the signal morphogen, received from the } \\
& \text { dermis. }
\end{aligned}
$$

(Morphogen variables and related constants specific to the epidermal layer are distinguished from those of the dermal layer by using the hat symbol.)

The model equations describing the chemical interaction are

$$
\begin{aligned}
& \frac{\partial \hat{e}}{\partial t}=\overbrace{\hat{D_{e} \nabla^{2} \hat{e}}}^{\text {diffusion }}+\overbrace{f(\hat{N}, \hat{s})}^{\text {production }}-\overbrace{P_{e}(\hat{e}-e)}^{\text {dermal signal }}-\overbrace{\hat{\gamma} \hat{e}}^{\text {degradation }}, \\
& \frac{\partial s}{\partial t}=\overbrace{D_{s} \nabla^{2} s}^{\text {secretion }}+\overbrace{g(n, e)}^{\text {epidermal signal }}-\overbrace{P_{s}(\hat{s}-s)}^{\text {degradation }}-\overbrace{v s}^{\text {metabolism }}, \\
& \frac{\partial e}{\partial t}=\overbrace{D_{e} \nabla^{2} e}^{\text {diffusion }}+P_{e}(\hat{e}-e)-\overbrace{\text { metabolism }}^{\text {diffusion }}, \\
& \frac{\partial \hat{s}}{\partial t}=\overbrace{\hat{D_{s}} \nabla^{2} \hat{s}}^{\text {dist }}+P_{s}(\hat{s}-s)
\end{aligned}
$$


Equations (A.1a) and (A.1c) are the conservation equations for the morphogen $e$ and $e$ in each layer, and similar equations, (A.1b) and (A.1d), hold for the morphogens $s$ and $\hat{s}$. In each equation we have assumed that the morphogen concentration changes due to Fickian diffusion and that it diffuses across the basal lamina which separates the dermis from the epidermis. During paracrine signalling the chemical molecules are degraded by enzymes and the positive constants $\hat{\gamma}$ and $v$ are measures of the degradation rates, respectively, of $\hat{e}$ in the epidermis and $s$ in the dermis. In the dermis the signalling molecules $e$ attach to the mesenchymal receptor cells and are metabolized by them with rate $\gamma$. It is assumed that this metabolism is proportional to the receptor cell density $n$ and the chemical concentration $e$. Similarly, we assume that $s$ is metabolized by cells in the epidermal layer with rate $\hat{v}$.

The full model then consists of the seven equations (2.1), (2.3), (2.4), and (A.1 a, b, c, d). The nondimensionalization of this full model is carried out in Appendix B. The resulting equations are still extremely complicated and too involved to lend themselves to useful mathematical analysis. We therefore consider a reduced model by introducing a quasi-steady-state hypothesis.

We assume that the free morphogen concentration in any layer stays constant with time-so $\hat{e}, e, \hat{s}$, and $s$ are at their equilibrium states. This implies that the signalling morphogens are used up, degrade, and diffuse to another layer at the same rate they diffuse into, and are produced by, a layer. This assumption is consistent with the concept of paracrine signalling.

Furthermore, in paracrine signalling the chemical molecules are so rapidly metabolized by receptor cells or immobilized by extracellular enzymes that they only act on cells in their immediate neighbourhood. Since these two processes are apparently more important than local diffusion, we isolate them and as a result can simplify our system considerably by setting the parameters $\hat{D}_{e}, \hat{D}_{s}, D_{s}$, and $D_{e}$ to zero.

We thus have a quasi-steady-state model in which equations (A.1) reduce to four algebraic equations. Eliminating $s$ and $\hat{e}$ from these equations we can solve for $\hat{s}$ and $e$ as functions of $N$ and $n$.

We assume for simplicity that the production of both morphogens $\dot{e}$ and $s$ are proportional to the respective cell densities $N$ and $n$, so that

$$
f(N, s)=k_{e} N \quad \text { and } \quad g(n, e)=k_{s} n,
$$

where the positive constants $k_{e}$ and $k_{s}$ are the dimensionless epidermal and dermal production rates, respectively.

The above assumptions lead to the following functional forms for $s$ and $e$ in terms of $n$ and $N$ :

$$
\xi(N, n)=\frac{P_{s} k_{s} n}{v P_{s}+\left(v+P_{s}\right) \hat{v} N}, \quad e(N, n)=\frac{P_{e} k_{e} N}{\hat{\gamma} P_{e}+\left(\hat{\gamma}+P_{e}\right) \gamma n}
$$

Using the quasi-steady-state assumption, the dependence of $\tau$ on $\delta$ in (2.2) can be rewritten in terms of $n$ and $N$. Similarly the dependence of $\alpha$ on $e$ in (2.5) can be written in terms of $n$ and $N$.

Substituting (A.2) into expressions (2.2) and (2.5) and reducing the number of 
parameters by the substitutions

$$
\tau^{*}=\frac{\tau k_{s}^{2}}{v^{2}}, \quad c^{*}=\frac{c k_{s}^{2}}{v^{2}}, \quad \hat{v}^{*}=\frac{\nu\left(v+P_{s}\right)}{v P_{s}}, \quad \alpha^{*}=\frac{\alpha k_{e}}{\hat{\gamma}}, \quad \gamma^{*}=\frac{\gamma\left(\hat{\gamma}+P_{e}\right)}{\hat{\gamma} P_{e}}
$$

we get the interactions terms as shown in (2.6) and (2.7), where the asterisks have been dropped for notational convenience.

\section{Appendix B}

\section{Nondimensionalization}

To nondimensionalize the full tissue interaction model equations (2.1), (2.3), (2.4), and (A.1), we use the following procedure. Let $T_{0}$ and $L_{0}$ be typical time and length scales respectively, $N_{0}$ and $n_{0}$ typical cell densities and $s_{0}$ and $e_{0}$ typical morphogen concentrations. Define the dimensionless quantities

$$
\begin{gathered}
t^{*}=\frac{t}{T_{0}}, \quad x^{*}=\frac{x}{L_{0}}, \quad u^{*}=\frac{u}{L_{0}}, \quad N^{*}=\frac{N}{N_{0}}, \quad n^{*}=\frac{n}{n_{0}}, \\
s^{*}=\frac{s}{s_{0}}, \quad s^{*}=\frac{s}{s_{0}}, \quad \hat{e}^{*}=\frac{e}{e_{0}}, \quad e^{*}=\frac{e}{e_{0}}, \\
\mu_{1}^{*}=\frac{\mu_{1}(1+v)}{E T_{0}}, \quad \mu_{2}^{*}=\frac{\mu_{2}(1+v)}{E T_{0}}, \quad \tau^{*}\left(s^{*}\right)=\frac{\tau(s)(1+v)}{E}, \\
\left.\hat{D}_{e}^{*}=\frac{\hat{D}_{e} e_{0} T_{0}}{L_{0}^{2}}, \quad f^{*}\left(N^{*}, s^{*}\right)=\frac{\beta_{1}}{L_{0}^{2}}, \quad \beta_{2}^{*}=\frac{\beta_{2}}{L_{0}^{2}}, \quad \rho^{*}=\frac{\rho L_{0}^{2}}{E^{\prime}}, \quad s\right) \\
\hat{D}_{s}^{*}=\frac{\hat{D}_{s} s_{0} T_{0}}{L_{0}^{2}}, \quad P_{s}^{*}=P_{s} T_{0}, \quad \hat{v}=\hat{v} N_{0} T_{0}, \quad \hat{\gamma}^{*}=\hat{\gamma} T_{0}, \\
D^{*}=\frac{D T_{0}}{L_{0}^{2}}, \quad r^{*}=r n_{0}, \quad \alpha^{*}=\frac{\alpha T_{0}}{L_{0}^{2}}, \\
D_{s}^{*}=\frac{D_{s} s_{0} T_{0}}{L_{0}^{2}}, \quad g^{*}\left(n^{*}, e^{*}\right)=\frac{g(n, e)}{s_{0}}, \quad P_{s}^{*}=P_{s} T_{0}, \quad v^{*}=v T_{0}, \\
D_{e}^{*}=\frac{D_{e} e_{0} T_{0}}{L_{0}^{2}}, \quad P *=P_{e} T_{0}, \quad \gamma^{*}=\gamma n_{0} T_{0} .
\end{gathered}
$$

Introducing these quantities leads to the dimensionless system. For notational convenience we drop the asterisks.

\section{Acknowledgements}

GCC would like to thank the Rhodes Trust, Oxford, and the South African Foundation for Research Development for their financial support. This work 
(JDM) was in part supported by a grant (DMS-90039) from the US National Science Foundation.

This article was based on a paper read at the IMA Conference on the Mathematical Theory of the Dynamics of Biological Systems, held in Oxford, 1-3 July 1992.

\section{REFERENCES}

Chuong, C.-M., \& Edelman, G. M. 1985a Expression of cell adhesion molecules in embryonic induction. I. Morphogenesis of nestling feathers. J. Cell Biol. 101, 1009-26.

Chuong, C.-M., \& Edelman, G. M. 1985b Expression of cell adhesion molecules in embryonic induction. II. Morphogenesis of adult feathers. J. Cell Biol. 101, 1027-43.

Cruywagen, G. C., \& MurRay, J. D. 1992 On a tissue interaction model for skin pattern formation. J. Nonlinear Sci. 2, 217-40.

DAvidson, D. 1983a The mechanism of feather pattern development in the chick. I. The time determination of feather position. J. Embryol. Exp. Morph. 74, 245-59.

Davidson, D. 1983b The mechanism of feather pattern development in the chick. II. Control of the sequence of pattern formation. J. Embryol. Exp. Morph. 74, 261-73.

Dhoualley, D. 1973 Dermo-epidermal interactions between birds and mammals: Differentiation of cutaneous appendages. J. Embryol. Exp. Morph. 30, 587-603.

DhoualLLy, D. 1975 Formation of cutaneous appendages in dermo-epidermal recombination between reptiles, birds and mammals. Wilhelm Roux Arc. EntwMech. Org. 177, 323-40.

Dhouallly, D., \& Maderson, P. F. A. 1984 Ultrastructural observations on the embryonic development of the integument of Lacerta muralis (Lacertilia, Reptilia). J. Morph. 179, 203-28.

Edelman, G. M. 1986 Cell adhesion molecules in the regulation of animal form and tissue pattern. Ann. Rev. Cell Biol. 2, 81-116.

Edmund, A. G. 1969 Dentition. In: Biology of the Reptilia. (A. d'A. Bellairs \& T. S. Parsons, eds.). London: Academic Press.

Gallin, W. J., Chuong, C.-M., Finkel, L. H., \& Edelman, G. M. 1986 Antibodies to liver cell adhesion molecules perturb inductive interactions and alter feather pattern and structure. Proc. Natl. Acad. Sci. USA 83, 8235-9.

Grumer, M., \& Edelman, G. M. 1988 Neuron-glia cell adhesion molecules interact with neurons and astroglia via different binding mechanisms. J. Cell Biol. 106, 487-503.

Landau, L. D., \& LifshitZ, E. M. 1970 Theory of Elasticity, 2nd edn. New York: Pergamon.

Maderson, P. F. A. 1965a The embryonic development of the squamate integument. Acta Zool. 46, 275-95.

MADERSON, P. F. A. 1965b The structure and development of the squamate epidermis. In: Biology of the Skin and Hair Growth (A. G. Lyne \& B. F. Short, eds.). Sydney: Angus and Robertson.

MurRay, J. D. 1989 Mathematical Biology. Heidelberg: Springer.

Murray, J. D., Deeming, D. C., \& Ferguson, M. W. J. 1990 Size dependent pigmentation pattern formation in embryos of Alligator mississippiensis: Time of initiation of pattern generation mechanism. Proc. R. Soc. Lond. B 239, 279-93.

MURRAY, J. D., \& OsteR, G. F. 1984a Generation of biological pattern and form. IMA J. Math. Appl. Med. Biol. 1, 61-75.

MurRay, J. D., \& Oster, G. F. 1984 b Cell traction models for generating pattern and form in morphogenesis. J. Math. Biol. 19, 265-79.

MYERSCOUGH, M. R., \& MURRAY, J. D. 1990 Analysis of propagating pattern in a chemotaxis system. Bull. Math. Biol. 54(1), 77-94.

NAGORCKA, B. N. 1986 The role of a reaction-diffusion system in the initiation of skin organ primordia. I. The first wave of initiation. J. Theor. Biol. 121, 449-75.

Nagorcka, B. N., Manoranjan, V. S., \& Murray, J. D. 1987 Complex spatial patterns from tissue interactions-An illustrative model. J. Theor. Biol. 128, 359-74. 
Oster, G. F., \& Murray, J. D. 1989 Pattern formation models and developmental constraints. J. Exp. Zool. 251, 186-202.

Pearson, M., \& Elsdale, T. 1979 Somitogenesis in amphibian embryos. I. Experimental evidence for an interaction between two temporal factors in the specification of the somite pattern. J. Embryol. Exp. Morph. 51, 27-50.

R.AWLES, M. 1963 Tissue interactions in scale and feather development as studied in dermal epidermal recombinations. J. Embryol. Exp. Morph. 11, 765-89.

Shaw, L. J., \& Murray, J. D. 1990 Model for complex skin patterns. SIAM J. Appl. Math. $50(2), 628-48$.

Sengel, P. 1976 Morphogenesis of Skin. Cambridge University Press.

Wessells, N. K. 1977 Tissue Interaction in Development. Menlo Park, CA: Benjamin.

WolperT, L. 1981 Positional information and pattern formation. Phil. Trans. R. Soc. Lond. B 295, 441-50. 